\title{
Efeito do Tamanho de Partícula e do Nível de Substituição nos Valores Energéticos da Farinha de Carne e Ossos para Pintos de Corte ${ }^{1}$
}

\author{
Irineu Brugalli², Luiz Fernando Teixeira Albino ${ }^{3}$, Dirceu Jorge da Silva ${ }^{3}$, Paulo Cezar Gomes ${ }^{3}$, \\ Horacio Santiago Rostagno ${ }^{3}$, Martinho de Almeida e Silva ${ }^{4}$
}

\begin{abstract}
RESUMO - O objetivo deste trabalho foi determinar a energia metabolizável aparente (EMA) e aparente corrigida (EMAn) da farinha de carne e ossos (FCO) com diferentes granulometrias (grossa, média e fina), em dois níveis de substituição (20 e $40 \%$ ) para pintos de corte. Duzentos e oitenta pintos de corte com 15 dias de idade foram distribuídos em delineamento inteiramente casualizado, com sete tratamentos (seis tratamentos com farinha de carne e ossos e uma dieta referência), quatro repetições e dez aves por unidade experimental. Os valores médios de EMA para os níveis 20 e 40\% de substituição foram 2,60 e 2,27 e para EMAn, 2,50 e 2,14 kcal/g MS, respectivamente. Os resultados, considerando-se as granulometrias grossa, média e fina da FCO, foram 2,13; 2,65; e 2,53 kcal/g MS para EMA e 2,03; 2,56; e 2,37 kcal/g MS para EMAn, respectivamente.
\end{abstract}

Palavras-chave: energia metabolizável aparente, farinha de carne e ossos, pintos, tamanho de partículas

\section{Effect of the Particle Size and the Substitution Level on the Energy Values of Meat and Bone Meal for Broiler Chicks}

\begin{abstract}
The objective of this work was to determine the apparent metabolizable energy (AME) and nitrogen-corrected apparent metabolizable energy (AMEn) of meat and bone meal (MBM) with different particle sizes (coarse, medium and fine), in two levels of substitution ( 20 and 40\%) for broiler chicks. Two hundred and eighty broiler chicks with 15 days of age were allotted to a completely randomized design, with seven treatments (six treatments with meat and bone meal and a reference diet), four replicates and ten birds per experimental unit. The AME mean values for the 20 and $40 \%$ of substitution were 2.60 and 2.27 and the EMAn 2.50 and $2.14 \mathrm{Kcal} / \mathrm{g} \mathrm{DM}$, respectively. The results, considering coarse, medium and fine particle sizes of the MBM, were $2.13,2.65$ and $2.53 \mathrm{kcal} / \mathrm{g} \mathrm{DM}$ for AME, and 2.03, 2.56 and $2.37 \mathrm{kcal} / \mathrm{g}$ DM to AMEn, respectively.
\end{abstract}

Key Words: apparent metabolizable energy, meat and bone meal, broiler chicks, particle size

\section{Introdução}

A composição química dos alimentos é um dos fatores que determina seu valor nutricional. Assim, tornase importante avaliar o conteúdo e a disponibilidade dos nutrientes, bem como o valor energético desses alimentos, a fim de que haja melhor precisão na formulação e no balanceamento das rações para aves, tornando-as de menor custo. No cálculo de rações para aves, a energia metabolizável é a forma mais utilizada para expressar a energia disponível nos alimentos.

No Brasil, já existem tabelas de composição de alimentos (ROSTAGNO et al., 1983; EMBRAPA, 1991), mas, ainda, utilizam-se dados de composição dos alimentos oriundos de tabelas estrangeiras na formulação de rações balanceadas para aves (RHODIMET, 1993b; NRC, 1994). Assim, pode ocorrer variações na composição de alguns alimentos nacionais, quando comparada à composição apresentada em tabelas estrangeiras. Vários estudos têm sido conduzidos no sentido de conhecer melhor a composição química e energética dos alimentos nacionais (COLNAGO et al., 1979; FRANQUEIRA et al., 1979; e ALBINO et al., 1981, 1982, 1986, 1989). Entretanto, poucos trabalhos têm sido feitos para avaliar o efeito do tamanho de partículas sobre o valor energético dos alimentos utilizados em dietas para aves.

Trabalhando com suínos em crescimento e terminação, LIMA et al. (1990) avaliaram o efeito do farelo de girassol com moagem fina e moagem grossa na digestibilidade dos nutrientes e verificaram aumentos de 22,18 e $16 \%$ nos valores de matéria seca digestível, energia metabolizável e energia digestível, respectivamente, quando utilizado o farelo

\footnotetext{
${ }^{1}$ Parte da Tese apresentada à UFV pelo primeiro autor para obtenção do título de Mestre em Zootecnia.

2 Eng. Agrônomo, Estudante de Pós-Graduação em Zootecnia - UFV, Viçosa, MG - 36571-000, Bolsista - CNPq.

${ }^{3}$ Professor do Departamento de Zootecnia-UFV, Viçosa, MG - 36571-000, Bolsista - CNPq.

${ }^{4}$ Professor da UENF.
} 
de girassol com moagem fina. Entretanto, DOUGLAS et al. (1990), LOTT et al. (1992) e NIR et al. (1994a), trabalhando com frangos de corte, e LIMA et al. (1990) e ZANOTTO (1995b), com suínos, observaram efeitos benéficos da diminuição do tamanho de partículas dos ingredientes no desempenho animal. Contudo, NIR et al. (1990, 1994b) encontraram melhores resultados de desempenho com o uso de partículas mais grossas. Já REECE et al. (1986) e ZANOTTO et al. (1994) não encontraram efeito do grau de moagem do milho sobre o desempenho de frangos de corte.

Os alimentos em teste normalmente substituem 30 ou $40 \%$ a ração-referência em ensaio biológico pelo método tradicional (coleta total de excretas). Trabalhando com níveis que variaram de 5 a $60 \%$ de substituição, LESSIRE et al. (1985), PESTI et al. (1986), MARTOSISWOYO e JENSEN (1988) e DOLZ e DE BLAS (1992) demostraram que os valores de energia matabolizável da farinha de carne e ossos variaram conforme o nível de substituição, tendo valores significativamente maiores com os menores níveis de substituição.

O presente trabalho foi realizado com o objetivo de avaliar o efeito do tamanho das partículas e do nível de substituição sobre os valores de energia metabolizável da farinha de carne e ossos para pintos de corte.

\section{Material e Métodos}

O experimento foi conduzido, em baterias frias, no Laboratório de Animais do Departamento de Zootecnia (DZO) do Centro de Ciências Agrárias da Universidade Federal de Viçosa, com duração de dez dias, sendo cinco dias de adaptação e cinco dias de coleta total de excretas. As temperaturas médias máximas e mínimas foram 23,6 e $20,2^{\circ} \mathrm{C}$, respectivamente, e a umidade relativa média do ar foi de $68,9 \%$.

Foram utilizados 280 pintos Hubbard, sendo metade de cada sexo, com 15 dias de idade, em delineamento experimental inteiramente ao acaso, em esquema fatorial $3 \times 2$ (granulometria x nível), com sete tratamentos (seis rações-teste e uma ração-referência), quatro repetições e dez aves por unidade experimental, sendo cinco machos e cinco fêmeas.

O alimento estudado foi a farinha de carne e osso (FCO) com três diferentes diâmetros geométricos médios de partículas: grosso $(0,59 \mathrm{~mm})$, médio $(0,51 \mathrm{~mm})$ e fino $(0,42 \mathrm{~mm})$, denominados granulometria grossa, média e fina, respectivamente. Os valores granulométricos médios foram obtidos por meio do uso de peneiras com diferentes aberturas.

As análises físico-químicas dos ingredientes foram realizadas no Laboratório de Nutrição Animal do DZO da UFV. As análises de composição química dos ingredientes da ração-referência foram feitas segundo SILVA (1990) e os valores energéticos obtidos de ROSTAGNO et al. (1983). As análises granulométricas, a digestibilidade em pepsina e a acidez da FCO foram realizadas de acordo com as normas do LABORATÓRIO NACIONAL DE REFERÊNCIA ANIMAL-LANARA (1981). A raçãoreferência, à base de milho e farelo de soja, suplementada com vitaminas e minerais e formulada para atender às exigências nutricionais de pintos de corte na fase inicial, de acordo com ROSTAGNO (1990), é apresentada na Tabela 1.

Os sete tratamentos utilizados na determinação da energia metabolizável da FCO consistiram de uma ração-referência e seis rações-teste. As rações-teste foram obtidas mediante substituição da ração-referência, em base de matéria natural, por dois níveis (20 e $40 \%$ ) de FCO e, em cada nível de substituição, com três granulometrias da FCO (grossa, média e fina).

A água e as rações foram fornecidas à vontade por dez dias, sendo cinco para adaptação às rações experimentais e ao novo ambiente e cinco para coleta total de excretas de cada unidade experimental, em intervalos de 12 horas. As rações experimentais foram pesadas no início e no final do período experimental (20 - 25 dias de idade dos pintos), para se determinar o consumo.

Para as coletas das excretas, foram utilizadas bandejas da própria bateria, cobertas com plástico, colocadas sob cada compartimento, para individualizar o material e evitar perdas. O material recolhido, colocado em sacos plásticos, foi pesado e armazenado em congelador até o período final de coleta. Ao final do período de coleta, as amostras foram descongeladas, reunidas por repetição e homogeneizadas. Após, foram retiradas alíquotas de aproximadamente $400 \mathrm{~g}$, que foram levadas à estufa ventilada, à temperatura de $55^{\circ} \mathrm{C}$ por 48 horas para secagem e, só então, foram moídas e acondicionadas em frascos de vidro vedados para as análises de matéria seca, energia bruta e nitrogênio, segundo SILVA (1990). Amostras das rações também foram retiradas para análises.

Determinados os valores de matéria seca, energia bruta e nitrogênio das rações e das excretas, calcularam-se os valores de energia metabolizável aparente (EMA) do alimento, por meio de equações, segundo MATTERSON et al. (1965), os quais foram ajustados com base na retenção de nitrogênio, obtendo-se os 
Tabela 1 - Composição percentual da ração-referência

Table 1 - Percentage composition of the reference diet

\begin{tabular}{|c|c|}
\hline \multicolumn{2}{|l|}{ Ingredient } \\
\hline Milho (Corn) & 51,101 \\
\hline Farelo de soja (Soybean meal) & 41,542 \\
\hline Óleo vegetal (Vegetal oil) & 3,444 \\
\hline P bicálcico (Dicalcium P) & 1,805 \\
\hline Calcário (Limestone) & 1,168 \\
\hline $\operatorname{Sal}($ Salt) & 0,350 \\
\hline Mistura mineral (Mineral premix) ${ }^{1}$ & 0,050 \\
\hline Mistura vitamínica (Vitamin premix) ${ }^{2}$ & 0,100 \\
\hline DL-Metionina (DL-Methionine) $99 \%$ & 0,180 \\
\hline Cloreto de colina (Choline chloride) $60 \%$ & 0,100 \\
\hline Bacitracina de zinco (Zinc bacitracin) $10 \%$ & 0,050 \\
\hline BHT & 0,010 \\
\hline \multicolumn{2}{|l|}{ Valores calculados } \\
\hline \multicolumn{2}{|l|}{ Calculated values } \\
\hline Proteína bruta (Crude protein), $\%$ & 22,000 \\
\hline $\mathrm{EM}(M E) \mathrm{kcal} / \mathrm{kg}$ & $3.000,000$ \\
\hline Metionina (Methionine), \% & 0,535 \\
\hline Met. + Cis. $(\%)$ & 0,914 \\
\hline Lisina (Lysine), \% & 1,310 \\
\hline $\mathrm{Ca}(\%)$ & 1,000 \\
\hline $\mathrm{P}$ disponível (Available P) \% & 0,454 \\
\hline \multicolumn{2}{|c|}{ 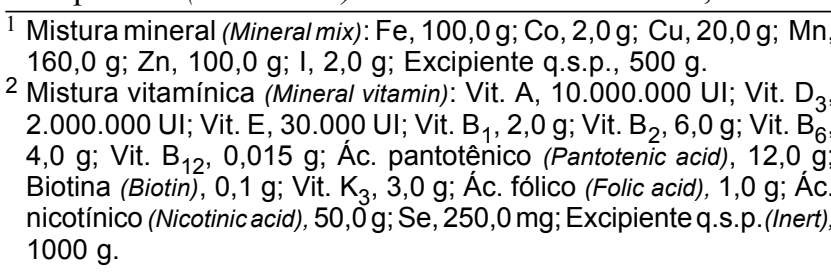 } \\
\hline
\end{tabular}

valores de energia metabolizável aparente corrigida pelo balanço de nitrogênio (EMAn).

As análises estatísticas dos dados foram realizadas utilizando o programa SAEG (Sistemas para Análises Estatísticas e Genéticas) da UNIVERSIDADE FEDERAL DE VIÇOSA-UFV (1982). A comparação entre médias dos tratamentos foi feita pelo teste Newman-Keuls.

\section{Resultados e Discussão}

Constata-se que a farinha de carne e ossos obtida neste trabalho, em relação às apresentadas nas tabelas de composição de alimentos (ROSTAGNO et al., 1983; EMBRAPA, 1991; RHODIMET, 1993b; e NRC, 1994), apresentou grande variação em sua composição química, principalmente em seu teor de gordura e minerais. Por ser produto resultante do processamento de resíduos de abatedouro, atribui-se esta variação ao uso de diferentes proporções desses resíduos, além dos diferentes métodos de processamento empregados.

Os valores de energia metabolizável aparente (EMA) e aparente corrigida (EMAn) da FCO com diferentes granulometrias e nível de substituição são apresentados nas Tabelas 2 e 3 .

Os valores obtidos de EMA e EMAn foram maiores $(\mathrm{P}<0,01)$ com $20 \%$ que $40 \%$ de substituição, estando de acordo com os trabalhos de LESSIRE et al. (1985), PESTI et al. (1986), MARTOSISWOYO e JENSEN (1988) e DOLZ e DE BLAS (1992), que atribuem a redução dos valores energéticos com aumento do nível de substituição à interferência dos altos níveis de cálcio com a absorção de gordura, à diminuição na absorção de ácidos graxos com o aumento da relação ácidos graxos saturados:insaturados, ao aumento no imbalanço de aminoácidos, à diminuição na digestibilidade da proteína da FCO causada pelo alto conteúdo de minerais e à redução no consumo, ocasionando maior interferência das perdas de energia fecal metabólica e urinária endógena.

Portanto, os valores de energia metabolizável seriam subestimados, se as estimativas obtidas com altos níveis de substituição (30 e 40\%) fossem extrapoladas para os níveis comumente usados em dietas práticas, que são menores que $10 \%$. Aparentemente estes dados recomendariam, portanto, a medição da energia metabolizável da FCO em rações contendo níveis práticos desse ingrediente. No entanto, POTTER (1972) aponta o nível de substituição do ingrediente-teste como fonte de variação, mostrando que, quanto maior o nível de substituição, menor a variação nos resultados obtidos.

Com a correção dos valores de EMA pela retenção de nitrogênio, verificou-se redução nos valores de EMAn, em razão do balanço positivo de nitrogênio, que, em geral, está de acordo com a literatura consultada. POTTER (1972) sugere a correção para retenção de nitrogênio zero, quando se comparam valores de energia metabolizável do mesmo alimento para aves de diferentes idades, uma vez que existem diferenças nas taxas de síntese ou catabolismo de proteínas.

Considerando o tamanho de partículas, houve diferença $(\mathrm{P}<0,05)$ nos valores de EMA e EMAn da FCO, obtendo-se valores menores $(\mathrm{P}<0,05)$ para a FCO grossa, em relação à FCO média e fina, que não diferiram entre si. Essas diferenças são atribuídas, em parte, à eficiência da digestão dos alimentos, que pode ser influenciada, entre outros fatores, pela superfície de exposição destes às ações das secreções digestivas, bem como pela taxa de passagem no trato gastrointestinal das aves (ZANOTTO et al., 1995a). 
Tabela 2 - Valores de energia metabolizável aparente (EMA) da farinha de carne e ossos com diferentes granulometrias, em dois níveis de substituição ${ }^{1,2}$

Table 2 - Apparent metabolizable energy values (AME) of meat and bone meal with different particle sizes, in two substitution levels ${ }^{1,2}$

\begin{tabular}{|c|c|c|c|}
\hline $\begin{array}{l}\text { Farinha de carne e ossos } \\
\text { Meat and bone meal }\end{array}$ & & & $\begin{array}{l}\text { Média } \\
\text { Mean }\end{array}$ \\
\hline & $20 \%$ & $40 \%$ & \\
\hline Granulometria & & & \\
\hline Particlesize & & & \\
\hline $\begin{array}{l}\text { Grossa }\left(\mathrm{DGO}^{4}=0,59 \mathrm{~mm}\right) \\
\text { Coarse }\end{array}$ & $2,37 \pm 0,31$ & $1,88 \pm 0,14$ & $2,13 \mathrm{~B}$ \\
\hline $\begin{array}{l}\text { Média }(\mathrm{DGO}=0,51 \mathrm{~mm}) \\
\text { Medium }\end{array}$ & $2,80 \pm 0,20$ & $2,49 \pm 0,35$ & $2,65 \mathrm{~A}$ \\
\hline $\begin{array}{l}\text { Fina }(\mathrm{DGO}=0,42 \mathrm{~mm}) \\
\text { Fine }\end{array}$ & $2,62 \pm 0,29$ & $2,44 \pm 0,28$ & $2,53 \mathrm{~A}$ \\
\hline Média (Mean) & $2,60 \mathrm{a}$ & $2,27 b$ & \\
\hline
\end{tabular}

Tabela 3 - Valores de energia metabolizável aparente corrigida (EMAn) da farinha de carne e ossos com diferentes granulometrias, em dois níveis de substituição ${ }^{1,2}$

Table 3 - Nitrogen-corrected apparent metabolizable energy values (AMEn) of meat and bone meal with different particles sizes, in two substitution levels ${ }^{1,2}$

\begin{tabular}{|c|c|c|c|}
\hline $\begin{array}{l}\text { Farinha de carne e ossos }{ }^{3} \\
\text { Meat and bone meal }\end{array}$ & & & $\begin{array}{l}\text { Média } \\
\text { Mean }\end{array}$ \\
\hline & $20 \%$ & $40 \%$ & \\
\hline Granulometria & & & \\
\hline Particlesize & & & \\
\hline $\operatorname{Grossa}\left(\mathrm{DGO}^{4}=0,59 \mathrm{~mm}\right)$ & $2,32 \pm 0,29$ & $1,74 \pm 0,12$ & $2,03 \mathrm{~B}$ \\
\hline Coarse & & & \\
\hline Média $(\mathrm{DGO}=0,51 \mathrm{~mm})$ & $2,69 \pm 0,19$ & $2,42 \pm 0,32$ & $2,56 \mathrm{~A}$ \\
\hline Medium & & & \\
\hline Fina $(\mathrm{DGO}=0,42 \mathrm{~mm})$ & $2,48 \pm 0,26$ & $2,26 \pm 0,23$ & $2,37 \mathrm{~A}$ \\
\hline Fine & & & \\
\hline Média (Mean) & $2,50 \mathrm{a}$ & $2,14 b$ & \\
\hline $\begin{array}{l}1 \text { Valores expressos em } \mathrm{kcal} / \mathrm{g} \\
2 \text { Médias, na linha/coluna, segui } \\
\text { Keuls }(P<0,05) \text {. } \\
{ }_{2}^{2} \text { Means, within a row/column, followe } \\
{ }^{3} \text { MS }(D M)=90,24 \% \text {. } \\
{ }^{4} \text { DGO = diâmetro geométrico (Gn }\end{array}$ & $\begin{array}{l}\text { cal/g DM). } \\
\text { diferentes sã } \\
\text { tters are differ }\end{array}$ & $\begin{array}{l}\text { ntes pelos } t \\
(P<.01) \text { and } N\end{array}$ & $\begin{array}{l}=0,01) \\
s(P<.0\end{array}$ \\
\hline
\end{tabular}

Sabe-se que o tempo de passagem do bolo alimentar pelo trato gastrointestinal das aves é relativamente curto, portanto, a redução do tamanho de partículas contribuiu substancialmente para melhor digestão e absorção dos nutrientes.

Considerando a média, os valores de EMA e EMAn da FCO variaram, consideravelmente, quando comparados aos das tabelas de composição de alimentos (ROSTAGNO et al., 1983; EMBRAPA, 1991; RHODIMET, 1993b; e NRC, 1994), provavelmente em virtude da utilização de diferentes proporções de matérias-primas empregadas e diferentes métodos de processamento para a obtenção desses alimentos.

\section{Conclusões}

O valor 2,59 $\mathrm{kcal}$ de EMAn/grama (na matéria seca) é o recomendado para efeito de cálculo, quando a FCO é utilizada em rações para aves, considerando o nível $20 \%$ de substituição e as granulometrias fina e média.

Em razão das diferenças encontradas nos valores energéticos da FCO, comparados aos citados na literatura, torna-se importante a padronização físico-química da FCO, a fim de se usarem valores energéticos mais exatos na formulação de rações para aves. 


\section{Referências Bibliográficas}

ALBINO, L.F.T., FERREIRA, A.S., FIALHO, E.T. et al. 1982. Determinação dos valores de energia metabolizável e matéria seca aparentemente metabolizável de alguns alimentos. $R$. Soc. Bras. Zootec., 11(2):207-221.

ALBINO, L.F.T., FIALHO, E.T., BLUME, E. 1986. Energia metabolizável e composição química de alguns alimentos para frangos de corte. R. Soc. Bras. Zootec., 15(3):184-193.

ALBINO, L.F.T., ROSTAGNO, H.S., FONSECA, J.B. et al. 1981. Tabela de composição de alimentos. V. Valores de composição química e de energia determinados com aves em diferentes idades. R. Soc. Bras. Zootec., 10(1):133-146.

ALBINO, L.F.T., RUTZ, F., BRUM, P.A.R. et al. 1989. Energia metabolizável aparente e verdadeira de alguns alimentos determinados com galos. Pesq. Agropec. Bras., 24(12):1433-1437.

COLNAGO, G.L., COSTA, P.M.A., FONSECA, J.B. et al. 1979. Composição química e energia metabolizável de alguns alimentos para poedeiras. R. Soc. Bras. Zootec., 8(4):654-664.

DOLZ, S., DE BLAS, C. 1992. Metabolizable energy of meat and bone meal from spanish rendering plants as influenced by level of substitution and method of determination. Poult. Sci., 71(2):316-322.

DOUGLAS, J.H., SULLIVAN, T.W., BOND, P.L. et al. 1990. Influence of grinding, rolling and pelleting on the nutritional value of grain sorghums and yellow corn for broilers. Poult. Sci., 69(12):2150-2156.

EMBRAPA - Centro Nacional de Pesquisa de Suínos e Aves. 1991. Tabela de composição química e valores energéticos de alimentos para suínos e aves. 3.ed. Concórdia. 97p. (EMBRAPA - CNPSA. Documentos, 19).

FRANQUEIRA, J.M., ROSTAGNO, H.S., SILVA, D.J. et al. 1979. Tabela de composição de alimentos concentrados. III. Valores de composição química e de energia metabolizável determinados com poedeiras. R. Soc. Bras. Zootec., 8(4):697-708.

LABORATÓRIO NACIONAL DE REFERÊNCIA ANIMAL LANARA. Portaria n. 002 de 20 out. 1981. (Apostila).

LESSIRE, M., LECLERCQ, B., CONAN, L. et al. 1985. A methodological study of the relationship between the metabolizable energy values of two meat meals and their level of inclusion in the diet. Poult. Sci., 64(11):1721-1728.

LIMA, G.J.M.M., GOMES, P.C., BARIONI JR., W. et al. 1990. Tamanho da partícula afeta a digestibilidade do farelo de girassol em suínos. Concórdia: EMBRAPA-CNPSA. 2p. (EMBRAPA-CNPSA. Comunicado técnico, 90).

LOTT, B.D., DAY, E.J., DEATON, J.W. et al. 1992. The effect of temperature, dietary energy level, and corn particle size on broiler performance. Poult. Sci., 71(4):618-624.

MARTOSISWOYO, A.W., JENSEN, L.S. 1988. Available energy in meat and bone meal as measured by different methods. Poult. Sci., 67(2):280-293.

MATTERSON, L.D., POTTER, L.M., STUTZ, N.W. et al. 1965. The metabolizable energyoffeeds ingredient for chickens. Storrs: University of Connecticut - Agricultural Experiment Station. 11p. (Research Report, 7).

NATIONAL RESEARCH COUNCIL - NRC. 1994. Nutrient requeriments of poultry. 9.ed. Washington, D.C.: National Academic Press. 155p.

NIR, I., MELCION, J.P., PICARD, M. 1990. Effect of particle size of sorghum grains on feed intake and performance of young broilers. Poult. Sci., 69:2177-2184.

NIR, I., SHEFET, G., ARONI, Y. 1994a. Effect of particle size on performance: 1. Corn. Poult. Sci., 73(1):45-49.
NIR, I., HILLEL, R., SHEFET, G., NITSAN, Z. 1994b. Effect of grain particle size on performance. 2. Grain texture interactions. Poult. Sci., 73(6):781-791.

PESTI, G.M., FAUST,L.O., FULLER, H.L. et al. 1986. Nutritive value of poultry by-product meal. 1. Metabolizable energy values as influenced by method of determination and level of substitution. Poult. Sci., 65(12):2258-2267.

POTTER, L.M. 1972. The prevision of measuring metabolizable energy in poultry feedstuffs. Feedstuffs, 44(12):28-30.

REECE, F.N., LOTT, B.D., DEATON, J.W. 1986. Effects of environmental temperature and corn particle size on response of broilers to pelleted feed. Poult. Sci., 65(4):636-641.

RHODIMET nutrition guide. 1993. 2. ed. s.1.: Rhone-Poulenc Animal Nutrition. 55p.

ROSTAGNO, H.S. Valores de composição de alimentos e de exigências nutricionais utilizados na formulação de rações para aves. In: REUNIÃO ANUAL DA SOCIEDADE BRASILEIRA DE ZOOTECNIA, 27, 1990, Piracicaba, SP. Anais... Piracicaba: SBZ, 1990, p.11-30.

ROSTAGNO, H.S., SILVA, D.J., COSTA, P.M.A. et al. 1983. Composição de alimentos e exigências nutricionais de aves e suínos: tabelas brasileiras. Viçosa, MG: UFV. 61p.

SILVA, D.J. 1990. Análise de alimentos: métodos químicos e biológicos. 2. ed. Viçosa, MG: UFV. 165p.

UNIVERSIDADE FEDERAL DE VIÇOSA. 1982. Central de Processamento de Dados-UFV/CPD. SAEG-Sistema de análise estatística e genética. Viçosa. 59p.

ZANOTTO, D.L., ALBINO, L.T.F., BRUM, P.A.R. et al. Efeito do grau de moagem no valor energético do milho para frangos de corte. In: REUNIÃO ANUAL DA SOCIEDADE BRASILEIRA DE ZOOTECNIA, 31, 1994, Maringá. Anais... Maringá: SBZ, 1994. p.57.

ZANOTTO, D.L., MONTICELLI, C., MAZZUCO, H. Implicações da granulometria de ingredientes de rações sobre a produção de suínos e aves. In: SIMPÓSIO LATINOAMERICANO DE NUTRIÇÃO DE SUÍNOS E AVES, 1995, Campinas. Anais... Campinas: CBNA, 1995a. p.111-133.

ZANOTTO, D.L., NICOLAIEWSKY, S., FERREIRA, A.S. et al. 1995b. Granulometria do milho na digestibilidade das dietas para suínos em crescimento e terminação. R. Soc. Bras. Zootec., 4(3):428-436.
Recebido em: 06/11/98

Aceito em: 03/03/99 